\title{
A Benign Cultured Colon Adenoma Bears Three Genetically Altered Colon Cancer Oncogenes, but Progresses to Tumorigenicity and Transforming Growth Factor-Beta Independence without Inactivating the p53 Tumor Suppressor Gene
}

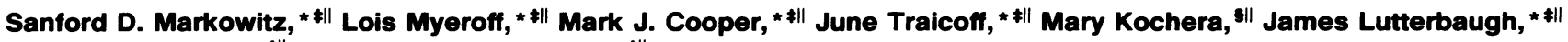 \\ Margaret Swiriduk, ${ }^{\star * \|}$ and James K. V. Willson * *\| \\ ${ }^{*}$ Ireland Cancer Center, Case Western Reserve University; and ${ }^{\ddagger}$ Division of Hematology-Oncology, Department of Medicine, and \\ ${ }^{\S}$ Center for Human Genetics, "University Hospitals of Cleveland, Cleveland, Ohio 44106
}

\begin{abstract}
We describe the spontaneous progression of a colon adenoma cell line to tumorigenicity and growth factor independence. This system allows direct comparison of biologic stages of malignant progression with alterations of colon cancer suppressor genes and oncogenes. VACO-235, a human colon adenoma cell line, is at early passages nontumorigenic in the nude mouse, unable to grow in soft agar, growth stimulated by serum and EGF, and growth inhibited by TGF-beta. VACO-235 daughter passages 93 and higher have in culture spontaneously progressed to being weakly tumorigenic, but retain all other growth characteristics of VACO-235 early passages. A mouse xenograft from late passage VACO-235 was reestablished in culture as the granddaughter cell line, VACO-411. VACO-411 is highly tumorigenic, clones in soft agar, and is unresponsive to serum, EGF, and TGF-beta. Early passage VACO-235 bears a mutant $\mathrm{K}$-ras allele, bears only mutant APC alleles, expresses no DCC transcripts, and expresses only wild type p53 transcripts. VACO411 retains the identical genotype, still expressing only wild type p53. Colonic cells after ras mutation, APC mutation, and DCC inactivation remain nontumorigenic and growth factor dependent. Malignant progression involves at least two additional steps, and in VACO-411 can proceed by a novel pathway not requiring p53 inactivation. (J. Clin. Invest. 1994. 93:10051013.) Key words: transforming growth factors $\bullet$ APC $\bullet$ DCC • $\mathrm{k}-$ ras $\bullet \mathbf{p 5 3}$
\end{abstract}

\section{Introduction}

To study the relationship between malignant progression and the primary genetic alterations in colon cancer, we have constructed a cell culture model of colon cancer progression. This model allows progressive genetic and phenotypic alterations during colon carcinogenesis to be directly compared. Colon carcinogenesis in humans proceeds through a multistep progression in which the normal colonic mucosa is progressively altered first to the benign but neoplastic colonic adenoma, then to increasingly advanced stages of invasive colon cancer (1). Multiple loci are targets for genetic alteration during colon carcinogenesis. These genes include K-ras, DCC, APC, and the

Address correspondence to Dr. Sanford Markowitz, UCRC \#2, Suite 200, 11001 Cedar Road, Cleveland, OH 44106.

Received for publication 30 March 1993 and in revised form 13 October 1993.

J. Clin. Invest.

(C) The American Society for Clinical Investigation, Inc. 0021-9738/94/03/1005/09 \$2.00

Volume 93, March 1994, 1005-1013 p53 gene (2-6). The K-ras gene is mutated in half of colon adenomas and half of colon carcinomas (2). DCC expression is generally absent in colon cancer, and deletion of one DCC allele is detected in half of colon adenomas and in $73 \%$ of colon carcinomas (2). Mutation of at least one APC allele is present in $60 \%$ of colon adenomas, and loss of both wild type APC alleles, by deletion and mutation, is present in $81 \%$ of all colon cancers $(6,7)$. Last, inactivation of the $p 53$ gene by combined deletion and mutation is detected in $70 \%$ of colon carcinomas (4).

This study presents a cell culture model of colon carcinogenesis. This model is based on three matched cell lines, VACO235ep (early passage), VACO-235lp (late passage), and VACO-411. These cell lines demonstrate increasing levels of biologic aggressiveness as assayed by tumorigenicity in the nude mouse, anchorage-independent growth in soft agar, resistance to the inhibitory growth factor TGF-beta, and independence from the growth requirement for stimulatory growth factors and/or serum. VACO-235, established from a premalignant human colonic villous adenoma (8), at early passages $(25-35)$ is nontumorigenic in the nude mouse, is unable to grow in soft agar, is growth stimulated by serum and EGF, and is growth inhibited by TGF-beta. Daughter passages 93 and later VACO-235 have in culture spontaneously progressed to being tumorigenic, causing tumor formation in half of nude mice by 2 mo after injection. Growth characteristics of late and early passage VACO-235 are in all other assays otherwise identical. A granddaughter cell line, VACO-411, was reestablished from one late passage VACO-235 nude mouse xenograft. VACO-411 is highly tumorigenic, forming within 1 wk tumors in $100 \%$ of all mice. VACO-411 additionally clones in soft agar, is unresponsive to growth stimulation by serum or EGF, and is unresponsive to growth inhibition by TGF-beta.

We have previously noted that APC is mutant at both alleles in early passage VACO-235, and remains so in late passage VACO-235 and in VACO-411 (6) (wherein VACO-411 is reported as VACO-410). Further genetic characterization of this model now reveals early passage VACO-235 bears a mutant K-ras allele, has deleted a DCC allele and does not express DCC transcripts, and expresses only wild type p53 transcripts. Thus in a colon epithelial cell model, combined ras mutation, APC mutation, and absent DCC expression accompany a neoplastic but benign phenotype. Unexpectedly, VACO-411 retains the identical genotype, including expressing only wild type p53 alleles. Progression to high grade tumorigenicity, growth factor independence, and anchorage independence requires at least two additional steps beyond ras mutation, APC mutation and loss of DCC expression. After the first step the neoplasm is tumorigenic, but cells remain growth factor dependent and incapable of soft agar growth. After the second step 
tumorigenicity is accelerated and cells are growth factor independent and clone in soft agar. Inactivation of the known p53 colon cancer suppressor gene is not a step on the pathway of progression to VACO-411. Progression to VACO-411 likely involves a novel genetic pathway. VACO-411 may be representative of the mechanism of progression in the subset of colon cancers that also retain wild type only p53 alleles.

\section{Methods}

Cell lines. VACO-235 early and late passages and VACO-411 were maintained on rat tail collagen-coated plates in MEM supplemented with $2 \%$ fetal bovine serum, insulin, transferrin, selenium, and hydrocortisone ( $\mathrm{MEM} 2{ }^{+}$media), as previously described (8).

Tumorigenicity. Tumorigenicity was assayed by subcutaneous injections of $5 \times 10^{6}$ cells into the flanks of BALB/c athymic female mice. Tumor formation was followed by weekly measurements with calipers.

Anchorage-independent growth. Anchorage-independent growth was assayed by cloning in soft agar as previously described $(9) .5 \times 10^{4}$ cells were seeded in a $35-\mathrm{mm}$ plate in $0.3 \%$ top agar over $0.6 \%$ bottom agar, each prepared in MEM $2^{+}$growth media. Colonies $>50$ cells in number were counted under a magnifying eyepiece weekly for $3 \mathrm{wk}$.

Growth studies. Cell lines were plated on fresh collagen-coated plates in MEM supplemented with hormones but without fetal bovine serum $\left(\mathrm{MEM}^{+}\right.$media $)$. Cells were plated in 24-well plates at $2 \times 10^{4}$ cells per well. For studies of positive growth factors, additions were made at $24 \mathrm{~h}$ of either fresh $\mathrm{MEM}^{+}$, fresh $\mathrm{MEM}^{+}$supplemented with EGF at $10 \mathrm{ng} / \mathrm{ml}$ (Sigma Chemical Co., St. Louis, MO), or MEM ${ }^{+}$ supplemented with $2 \%$ fetal bovine serum (GIBCO BRL, Gaithersburg, MD), and cells were counted after $7 \mathrm{~d}$ of growth. For studies of negative growth regulation, additions were made at $24 \mathrm{~h}$ of either $\mathrm{MEM}^{+}$supplemented with $10 \mathrm{ng} / \mathrm{ml} \mathrm{EGF}$ or of $\mathrm{MEM}^{+}$supplemented with $10 \mathrm{ng} / \mathrm{ml}$ EGF added to varying concentrations of TGF-beta. Cells were then counted after $6 \mathrm{~d}$ of growth. Cell counts were determined by removing the cells from the plates with EDTA and counting in a hemacytometer.

Subcloning VACO-235. VACO-235 mechanically disaggregated into single cells does not retain viability. However, clonal descendants from VACO-235 were derived, as previously reported, by transfecting VACO-235 with a control vector bearing only the sequences encoding G418 resistance, followed by selection in G418, and regrowth of single G418-resistant daughter colonies into cell lines (10).

Nucleic acids. RNA and DNA were prepared by extraction with guanidine isothiocyanate as previously described (11). Total cellular RNA and genomic DNA were separated by ultracentrifugation of the extract through a cesium cushion.

$K$-ras cloning and sequencing. The first exon of K-ras was cloned by PCR amplification from genomic DNA using previously published $5^{\prime}$ primer (GGCCTGCTGAAAATGACTGA) and 3' primer (GTCCTGCACCAGTAATATGC) (12). Amplification was for 30 cycles with denaturation at $94^{\circ} \mathrm{C}$ for $30 \mathrm{~s}$, annealing at $55^{\circ} \mathrm{C}$ for $30 \mathrm{~s}$, and extension at $72^{\circ} \mathrm{C}$ for $60 \mathrm{~s}$. PCR amplification products were separated from primers and nucleotides over a Chroma Spin-100 column (Clontech, Palo Alto, CA) and then directly sequenced using a Sequenase 2 kit as per the manufacturer's instructions (U.S. Biochem. Corp., Cleveland, $\mathrm{OH})$. The sequencing reaction was primed using the 3' PCR primer.

DCC analysis. DCC transcript was detected using the previously published exon connection RT-PCR assay $(3,13)$. PCR primer pairs included exon 1 (GATCTCTTGGACCGAATG) and exon 2 (GAAAGCTTTAATTTGAAAACCGG), exon 7(TTCCGCCATGGTTTTTAAATC) and exon 8 (AGCCTCATTTTCAGCCACACA), and exon 21 (CAGTGAGTGAAGGACCAA) and exon 23 (AGCAGCTAAACTTTGACATT). The PCR products were transferred to a nylon filter and detected by hybridization with, for the exon 1-2 product end-labeled oligonucleotide (TGAGTGCATGTGTGTGAGTGCTGCC), and for the exon 21-23 product end-labeled oligonucleotide
(GGTCACCCATCAGGACCACTCA). The exon 7-8 product was detected by hybridization with a random-primed partial DCC cDNA, clone DCC1.65, which was the kind gift of Dr. Bert Vogelstein (Johns Hopkins University, Baltimore, MD).

p53 cloning and sequencing. The complete coding sequence from the p53 transcript was cloned by RT-PCR using previously published PCR primers P1 and P2 (14). The PCR amplification products were cloned into bluescript, and pooled clones were sequenced following our previously published methods (4). Plasmid sequencing reactions used the Sequenase 2 sequencing kit as per the manufacturer's instructions (U.S. Biochem. Corp.).

p53 immunoblotting. Cell lines were lysed in $50 \mathrm{mM}$ Tris ( $\mathrm{pH} 8.0$ ), $120 \mathrm{mM} \mathrm{NaCl}, 0.5 \% \mathrm{NP}-40,5 \mathrm{mM}$ EDTA, $0.01 \mathrm{mg} / \mathrm{ml}$ aprotonin, $0.01 \mathrm{mg} / \mathrm{ml}$ leupeptin, $1 \mathrm{mM}$ PMSF, $30 \mathrm{mM}$ sodium tetrapyrophosphate, $50 \mathrm{mM}$ sodium fluoride, and $100 \mu \mathrm{M}$ sodium orthovanadate. 40 $\mu \mathrm{g}$ of lysate per sample was subjected to electrophoresis in an SDS polyacrylamide gel and transferred to BA-S nitrocellulose (Schleicher \& Schuell, Inc., Keene, $\mathrm{NH}$ ). The filter was blocked in $0.5 \%$ nonfat dried milk, probed with p53 monoclonal antibody 1801 (Oncogene Science, Manhasset, NY), and then incubated with horseradish peroxidase-conjugated secondary antibody and chemiluminescent substrates (Amersham Corp., Arlington Heights, IL).

Northern analyses. $10 \mu \mathrm{g}$ of total cellular mRNA was resolved by electrophoresis on agarose-formaldehyde gels (15). RNA was transferred to Nytran nylon membranes (Schleicher \& Schuell, Inc.) following the manufacturer's instructions. Double-stranded cDNA probes were labeled with ${ }^{32} \mathrm{P}$ by the random primer technique (16). Hybridization of probe to filters was at $42^{\circ} \mathrm{C}$ in $50 \%$ formamide, $5 \times$ SSPE, $2 \times$ Denhardts, $0.1 \%$ SDS, and $0.1 \mathrm{mg} / \mathrm{ml}$ denatured salmon sperm DNA. Final washes were in $0.1 \times \mathrm{SSC}, 0.1 \% \mathrm{SDS}$, at $65^{\circ} \mathrm{C}$. Blots were exposed on Kodak XAR film with an intensifying screen. The human p53 cDNA probe was excised from plasmid pC53-SN3 (10). The human MDM 2 cDNA was the kind gift of Dr. Bert Vogelstein. The glyceraldehyde-3-phosphate dehydrogenase probe is a PstI fragment of a chicken muscle cDNA (17). Blots hybridized to GAPDH underwent final washing at $56^{\circ} \mathrm{C}$.

Southern analyses. $10 \mu \mathrm{g}$ of genomic DNA from VACO-235 late passage, from VACO-411, and from an athymic BALB/c mouse were cut with restriction enzymes EcoRI or PstI. The DNA was resolved by agarose gel electrophoresis, and transferred to nitrocellulose membranes $(15,18)$. Blots were hybridized to probe a (SXpol), an 800-bp fragment from the Akv 623 murine retrovirus previously shown to detect most murine xenotropic retrovirus (19), or to probe b, a fulllength 8.2-kb clone of the MCF 247 murine retrovirus bearing substantial homology to the class of xenotropic retrovirus (20). SXpol was the kind gift of Dr. Christopher Thomas (University of Virginia, Charlottesville, VA), and MCF247 was the kind gift of Drs. John Coffin (Tufts University, Boston, MA) and Jonathan Stoye (National Institute for Medical Research, London, UK). Labeling of probe and hybridization of blots were as described above. Final washes were in $0.1 \times$ SSC, $0.1 \%$ SDS, at $60^{\circ} \mathrm{C}$.

\section{Results}

Spontaneous progression of the VACO-235 cell line. Early passages of the VACO-235 cell line (passages 25 and 35) in repeated trials were nontumorigenic when injected into nude mice. Fig. 1 shows the results of one such trial in which $5 \times 10^{6}$ VACO-235 cells, passage 35, were injected into the flanks of 10 mice. No tumor formation was observed during $90 \mathrm{~d}$ of observation. In separate trials of VACO-235 at passage 25 no tumor formation was observed during $130 \mathrm{~d}$ of observation postinjection. In contrast, after an additional $1 \mathrm{yr}$ of growth in culture VACO-235 spontaneously acquired weak tumorigenicity. Fig. 1 shows that at passage $123 \mathrm{VACO}-235$ was tumorigenic in 7 of 10 mice injected with a mean of 9-wk delay before the appearance of tumors of $200 \mathrm{~mm}^{3}$ volume (corresponding to a xeno- 


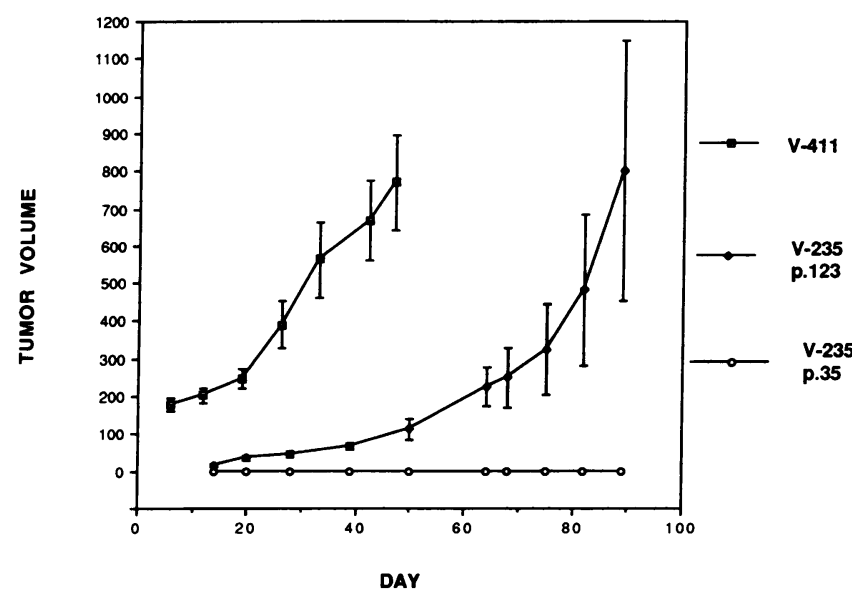

Figure 1. Xenograft formation by VACO cell lines. Shown are the mean tumor volumes of the xenografts formed after injection of 5 $\times 10^{6}$ cells at each of 10 injection sites for test cells VACO-235 passage 35 , VACO-235 passage 123 , and VACO-411. Error bars indicate the standard error of measurement of the mean xenograft volumes. VACO-411 formed xenografts at all 10 injection sites. VACO-235 passage 123 formed xenografts at 7 of 10 injection sites. VACO-235 passage 35 formed xenografts at no injection sites.

graft of size $8 \times 7(\mathrm{~mm})$. Similar weak tumorigenicity was demonstrable in VACO-235 as early as passage 85 . These data suggest that late passage VACO-235 (VACO-235lp) are a new daughter population that have in culture undergone a spontaneous progression event relative to the parental early passage VACO-235 (VACO-235ep). Conditions of growth in the nude mouse would likely select for yet additional progression events. To study such additional events a VACO-235 tumor xenograft, established from late passage VACO-235, was harvested and reestablished in culture as the now granddaughter cell line VACO-411. Fig. 1 demonstrates that the VACO-411 cell line is highly tumorigenic, giving rise within 2 wk in $100 \%$ of mice injected to tumors of $>200 \mathrm{~mm}^{3}$ volume. Histologic examination of VACO-235 and VACO-411 xenografts demonstrates (Fig. 2) that both are composed of similar highly mucinous gland-forming cells typical of adenocarcinomas (Fig. 2, insets). However, in the case of tumors formed by VACO-235 late passage cells, intracellular mucin is accompanied by a large lake of extracellular mucin around which the cells palisade. In contrast, xenografts formed by VACO-411 appear more cellular with little extracellular mucin noted. Surprisingly, despite the similarity both in gland formation and in individual cell cytology between the VACO-235- and VACO-411-derived xenografts, VACO-411 and VACO-235 in culture demonstrate markedly different morphologies and patterns of cell growth. Fig. 3 demonstrates that in culture both parental VACO-235ep and daughter VACO-235lp cell lines show marked epithelial features growing as epithelial islands of tightly apposed cells. Previous electron microscopy studies have demonstrated the expected tight junctions are present in these epithelial islands (8). In contrast, Fig. 3 shows that VACO-411 grows in culture as individual separate rounded cells. Thus the progression from VACO-235 late passage to VACO-411 is accompanied by a loss in tissue culture of such epithelial features as cell-to-cell adhesion. Interestingly, this difference in culture does not preclude VACO-411's forming in xenografts typical epithelial gland-like structures.
The presence of a VACO-411-like subpopulation within late passage VACO-235 could potentially account for the weak tumorigenicity displayed by late passage VACO-235. Morphologic examination gives no evidence of a VACO-411-like cell present in the late passage VACO-235 cultures. To further rule out such a possibility, late passage VACO-235 was recloned at passage 94 . The recloned line demonstrates both the epithelial morphology typical of VACO-235lp and again demonstrates the weak tumorigenicity typical of the uncloned VACO-235 late passage population (forming tumors at three of six injection sites at $8 \mathrm{wk}$ postinoculation). As the recloned line continues to be both epithelial and weakly tumorigenic, it is unlikely that the uncloned VACO-235lp is a mixture of a majority nontumorigenic cell (VACO-235ep-like) population along with a minority highly tumorigenic cell (VACO-411-like) population. Rather, we suggest late passage VACO-235 has acquired in culture an event permissive for xenograft growth, and VACO-411 has acquired subsequent additional progression events specifically selected during conditions of xenograft growth. Southern blot analysis for murine retroviral sequences rules out infection by a xenotropic murine retrovirus as an event participating in VACO-411 progression (S. Markowitz, unpublished data).

Acquisition of anchorage-independent growth during $V A C O-235$ progression. Concomitant with acquisition of tumorigenicity, VACO-411 also acquires capacity for anchorageindependent growth. Comparison in a soft agar cloning assay reveals that VACO-411 clones with $3.2 \%$ efficiency, whereas no soft agar clones are demonstrated when $5 \times 10^{4}$ VACO-235 early passage (passage 26) cells are inoculated into soft agar ( $<0.002 \%$ cloning efficiency). Examination of late passage VACO-235 (passage 91) also demonstrates no soft agar clones ( $<0.002 \%$ cloning efficiency). These data independently suggest VACO-411 does not preexist as a minor subpopulation within late passage VACO-235.

Acquisition of TGF-beta independence during VACO 235 progression. Loss of response to inhibition by the negative growth factor TGF-beta often accompanies carcinoma progression (21) and has been shown both to mark and to induce increased aggressiveness in colon cancer cell lines (22, 23). Fig. 4 demonstrates that the progressed line, VACO-411, is totally refractory to growth inhibition by TGF-beta. In contrast, Fig. 4 shows both early passage (passage 28 ) and late passage (passage 98) VACO-235 are $50 \%$ growth inhibited by $1 \mathrm{ng} / \mathrm{ml}$ TGFbeta and cease all growth when treated with $20 \mathrm{ng} / \mathrm{ml}$ TGFbeta. Thus, escape from TGF-beta responsiveness accompanies the progression of the VACO-235 colon adenoma cell line to anchorage independence and high grade tumorigenicity. And, in escaping control from inhibition by TGF-beta, VACO411 now resembles high grade cell lines previously established directly from colon cancers (22).

Acquisition of serum and TGF-alpha independence during $V A C O-235$ progression. Escape from growth dependence on serum or exogenous stimulatory hormonal growth factors is a feature distinguishing high grade, as opposed to low grade, colon cancer cell lines (22). Fig. 5 demonstrates that while early and late passage VACO-235 growth is stimulated by both serum and EGF, VACO-411 growth is independent of both serum and EGF. Indeed, growth of VACO-411 in basal media only is equal to maximal serum- or EGF-stimulated growth of early and late passage VACO-235 (Fig. 5). Thus, VACO-411 acquires a group of behaviors including high grade tumorigenic- 

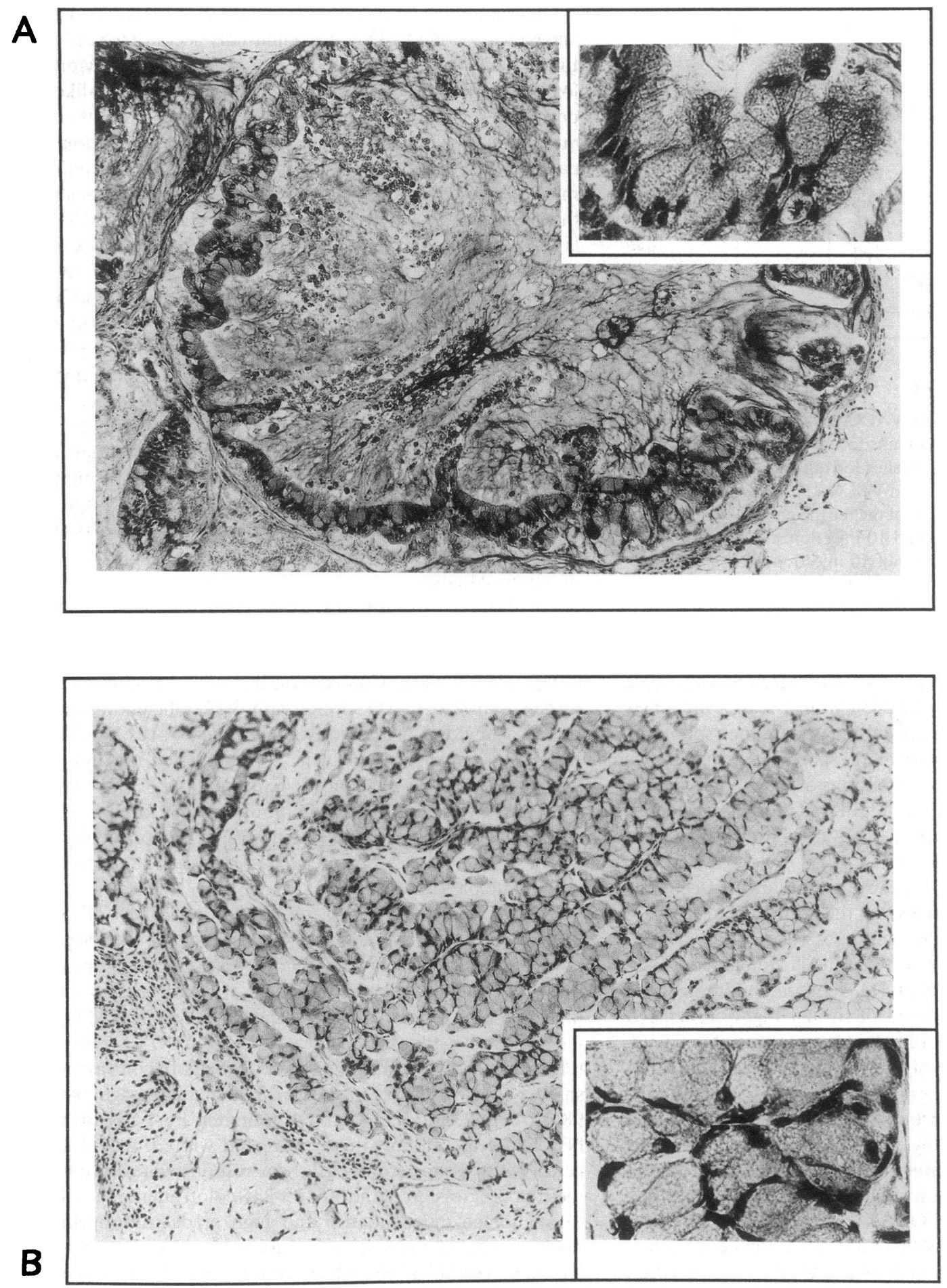

Figure 2. Morphology of VACO cell line xenografts. $(A)$ Histology of a representative xenograft grown from VACO-235 passage 93 cells. $(B)$ Histology of a representative xenograft grown from VACO-411 passage 10 cells $(\times 100$; insets, $\times 400)$. ity, anchorage-independent growth, and escape from regulation by both positive and negative growth factors.

$V A C O-235$ and $V A C O-411$ share common karyotypic features. Colon cancer progression is commonly accompanied by karyotypic evolution (2). Comparison of karyotypes from early passage VACO-235, late passage VACO-235, and VACO411 establishes all three lines are diploid with modal chromosome number 46 . Detailed cytogenetic study reveals that all three lines share common chromosomal alterations on chromosome 1 [del(1)(p36), dirins(1)(p36p31p36)], chromo- some $3[\operatorname{del}(3)(\mathrm{p} 21 \mathrm{p} 23)]$, chromosome $6[\operatorname{del}(6)(\mathrm{q} 21)$, $\mathrm{t}(6 ; 12)(\mathrm{q} 15 ; \mathrm{q} 22)]$, chromosome 7 [der(7)(q31.2)], chromosome 14 [del(14)(q11.2q24)], and chromosome 18 [del( 18)(q12.3q22)]. Fig. 6 displays three such marker chromosomes, 6, 14, and 18, and shows that in late passage VACO235 there is also addition of material added onto the terminus of the $6 p$ arm on the $t(6 ; 12)(q 15 ; q 22)$ translocation. This additional alteration is absent in VACO-235ep but is maintained in VACO-411. No further karyotypic alterations distinguish VACO-411 from late passage VACO-235. Thus, karyo- 

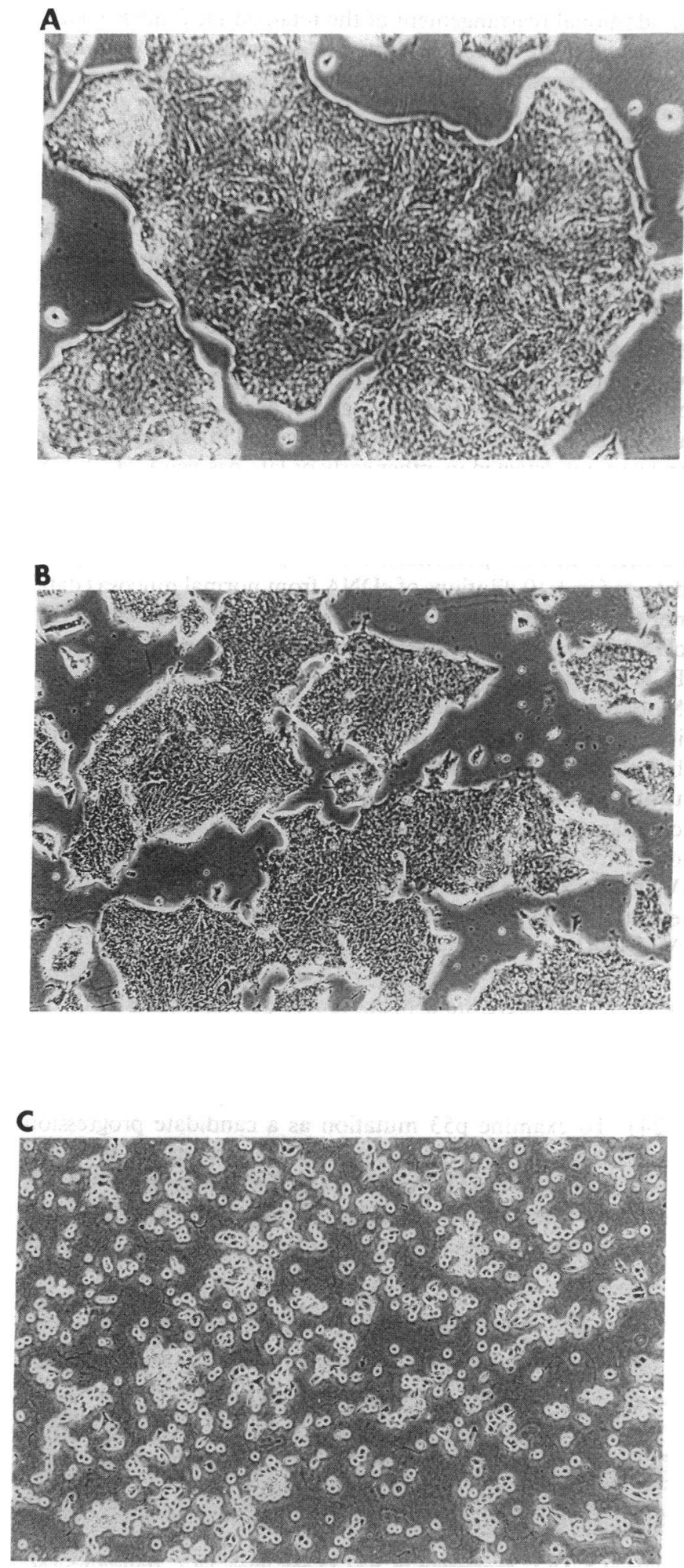

Figure 3. Morphology of VACO cell lines in culture. $(A)$ VACO-235 passage 29 ; $(B)$ VACO-235 passage 98 ; $(C)$ VACO-411 $(\times 100)$.

typic analysis confirms the derivation of VACO-411 from VACO-235 precursors, but does not pinpoint a candidate locus involved in progression to the VACO-411 phenotype.

Both VACO-235 and VACO-411 bear K-ras mutations. Mutant K-ras alleles are present in half of colon adenomas and half of colon carcinomas (2). K-ras mutations were analyzed

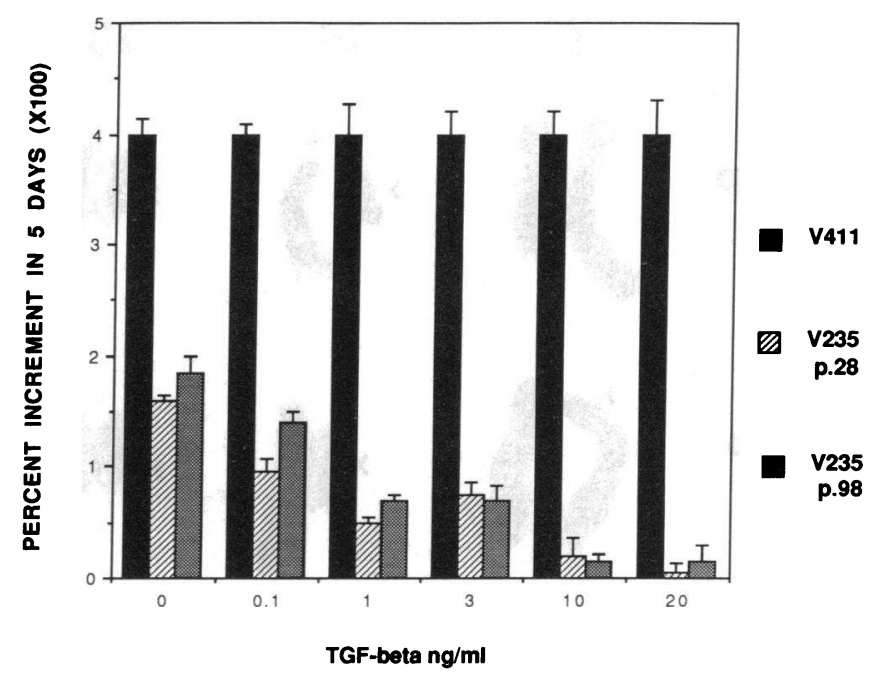

Figure 4. Growth inhibition of VACO cell lines by TGF-beta. VACO cell lines were seeded at $2 \times 10^{4}$ cells per well in serum-free growth media, and the next day TGF-beta was added. After $6 \mathrm{~d}$ of growth in TGF-beta, cells were counted and incremental cell growth was determined as: (final cell no. $-2 \times 10^{4}$ ) $/ 2 \times 10^{4}$. Experiments were performed in triplicate, and the means and standard error of the means are shown.

in VACO-235 and VACO-411 by PCR amplification of the $\mathrm{K}$-ras first exon and direct sequencing of the PCR product. An identical codon 13 GGC to GAC (gly to asp) mutation was detected in early and late passage VACO-235 and in VACO411. Retention of one wild type allele was also detected in each of the cell lines. Fig. 7 demonstrates the heterozygous wild type and mutant $\mathrm{K}$-ras alleles detected in VACO-235ep and retained in VACO-411, along with the wild type alleles found in a control cell line VACO-330. These data further confirm the derivation of VACO-411 from a VACO-235 precursor. Muta-

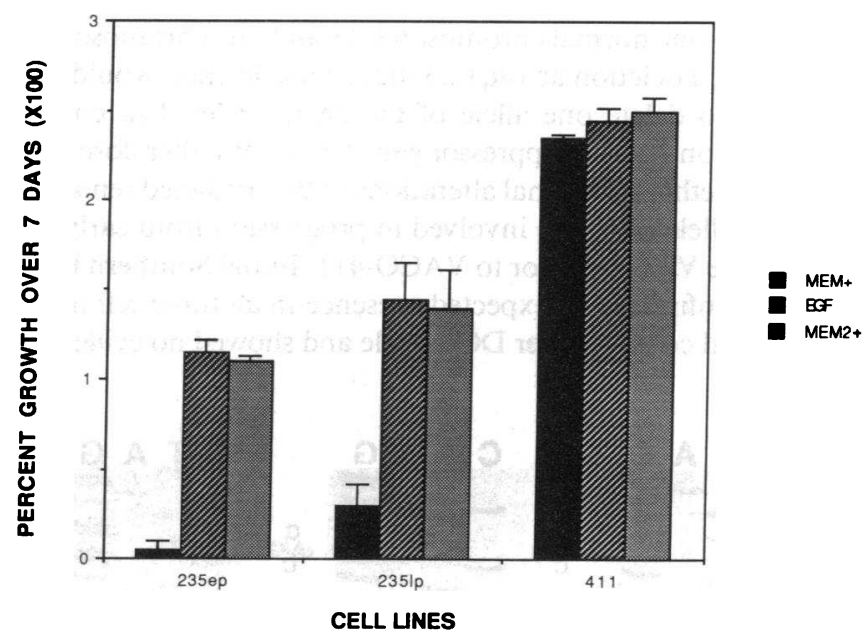

Figure 5. Growth response of VACO cell lines to serum and EGF. VACO cell lines were seeded at $2 \times 10^{4}$ cells per well in either $\mathrm{MEM}^{+}$ basal media, $\mathrm{MEM}^{+}$supplemented with EGF at $10 \mathrm{ng} / \mathrm{ml}$, or $\mathrm{MEM}^{+}$ supplemented with $2 \%$ fetal bovine serum $\left(\mathrm{MEM}^{+}\right)$. Cells were counted after $7 \mathrm{~d}$ of growth and incremental growth determined as: (final cell no. $-2 \times 10^{4}$ )/ $2 \times 10^{4}$. Experiments were performed in triplicate, and the means and standard error of the means are shown. 


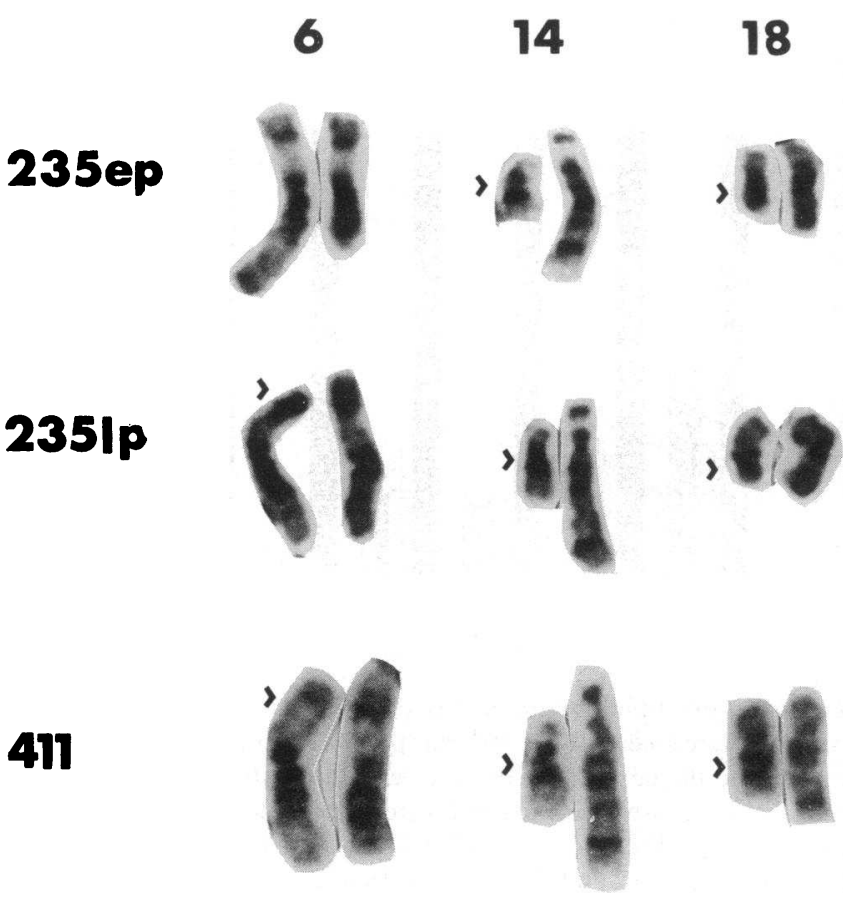

Figure 6. Partial karyotype of the VACO cell lines. Shown present in all the VACO cell lines are a marker chromosome 18 [del(18) (q12.3q22.2)] (arrow) and a marker chromosome 14 [del(14)(q11.2q24)] (arrow). A marker chromosome 6 $[\operatorname{der}(6) \mathrm{t}(6 ; 12)(\mathrm{q} 15 ; \mathrm{q} 22)]$ (left) is present in early passage VACO235. It is further identically altered in both late passage VACO-235 and in VACO-411 by addition of material at the tip of the short arm $[\operatorname{der}(6)(\mathrm{p} 22) \mathrm{t}(6 ; 12)(\mathrm{q} 15 ; \mathrm{q} 22)]$ (arrow). The remaining chromosome 6 is a del $(6)(q 21)$ in all three cell lines.

tion in K-ras is revealed as accompanying initial adenoma formation, and is not an additional event driving progression to late passage VACO-235 or to VACO-411.

$D C C$ gene expression is absent in early passage $V A C O$ 235 . VACO-411 shares in common with early and late passage VACO-235 one normal chromosome 18 and one chromosome 18 carrying a deletion at $18 \mathrm{q} 12.3-\mathrm{q} 22$. This deletion would be predicted to delete one allele of the DCC (deleted in colon cancer) colon cancer suppressor gene $(2,3)$. We therefore examined whether additional alterations of the predicted remaining DCC allele might be involved in progression from early to late passage VACO-235 or to VACO-411. Initial Southern blot analysis confirmed the expected presence in all three cell lines of a haploid copy number DCC allele and showed no evidence

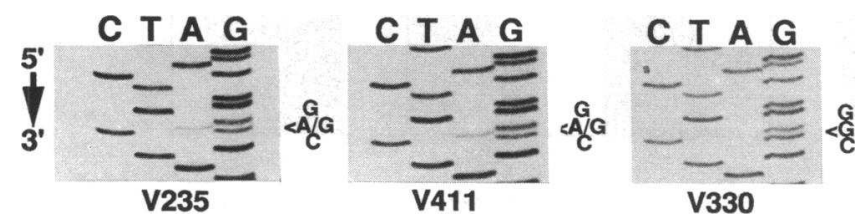

Figure 7. ras activation in VACO cell lines. Shown is the presence of a codon 13 gly (GGC) to asp (GAC) mutation in one allele of the K-ras gene in early passage VACO-235 and in VACO-411 as contrasted with the wild type K-ras sequence in the control line VACO-330. The gel was generated by direct dideoxy sequencing of the PCR-amplified $\mathrm{K}$-ras first exon. The sequence reads from top to bottom as the sequencing primer primes extension of the noncoding DNA strand. for additional rearrangement of the retained DCC allele (data not shown). We therefore elected to characterize in each cell message expression from the retained DCC allele. DCC expression was examined by an exon connection reverse PCR assay using a ${ }^{32} \mathrm{P}$-labeled oligonucleotide as a probe to detect the PCR product (3). Fig. 8 shows one such analysis using PCR primer pairs from DCC exons 1 and 2. DCC expression is easily detected in cDNA from three samples of normal colonic mucosa $(436 \mathrm{~N}, 442 \mathrm{~N}$, and $496 \mathrm{~N})$ and is absent in four colon cancer cell lines (SW480, VACO-206, VACO-429, and VACO-432). DCC expression is similarly absent in early passage VACO235, late passage VACO-235, and VACO-411. Similar results were obtained when the analysis was repeated using PCR primer pairs from DCC exons 7 and 8, or from DCC exons 21 and 23. DCC transcript was always detected in normal mucosa and was not detected in either early or late passage VACO-235 or VACO-411 (data not shown). We determined this assay would easily detect DCC expression at levels $10 \%$ of that in normal mucosa. Specifically, a PCR product could easily be detected in 1:10 dilutions of cDNA from normal mucosa (data not shown). As a positive control we determined that all the cDNAs compared produced an easily visualized signal when PCR was carried out with primers for the GAPDH transcript. Moreover, these $\mathrm{cDNAs}$ all produced a GAPDH PCR product in serial dilutions of from 1:1024-1:2048 (data not shown), but not at 1:4096. Hence, we judge these cDNAs are all within twofold concentration of one another and are reasonable to compare by the DCC PCR assay. Thus, we find that deletion of one DCC allele and absent DCC expression accompanies the VACO-235 early passage phenotype, and is not an additional event driving progression to late passage VACO-235 or VACO-411.

Expression of wild type p53 remains unaltered during $V A C O-235$ progression. Mutation of p53 correlates with the adenoma to carcinoma transition $(4,10)$, and mutant p53 alleles cloned from human colon cancers additionally will cooperate with mutant ras alleles in transforming rodent fibroblasts (24). To examine p53 mutation as a candidate progression event in the VACO-235 model, the p53 transcript was cloned by RT-PCR from cDNA from early and late passage VACO235 and from VACO-411. Unexpectedly, the p53 sequences

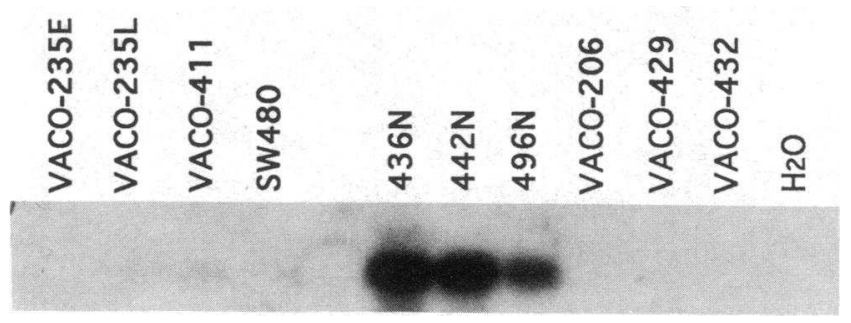

Figure 8. DCC expression in VACO cell lines. DCC transcript was detected by reverse PCR amplification using primers to DCC exons 1 and 2. The PCR-amplified product was transferred to a nylon filter and detected by hybridization with a ${ }^{32} \mathrm{P}$-labeled oligonucleotide probe. Shown is DCC expression in VACO-235 early passage ( $V A C O-235 E$ ), VACO-235 late passage ( $V A C O-235 L$ ), and VACO411. For comparison DCC expression is also shown in three samples of normal colonic mucosa $(436 \mathrm{~N}, 442 \mathrm{~N}$, and $496 \mathrm{~N})$ and in colon cancer cell lines SW480, VACO-206, VACO-429, and VACO-432. Also shown is a water blank carried through the assay as a negative control. 
were determined to be wild type in all three cell lines, VACO235 early passage, VACO-235 late passage, and VACO-411 (data not shown). In this analysis p53 sequences were run side by side on the same gel, a technique that allows easy recognition of even heterozygous mutant alleles (L. Myeroff, unpublished results). Western blot analysis with anti-p53 antibody 1801 also supports that only wild type p53 protein is present in early passage VACO-235, late passage VACO-235, and VACO411. Mutant p53 proteins typically display marked increases both in p53 protein half-life and in the accumulated level of total intracellular p53 protein (24-26). The panreactive antip53 antibody 1801 thus typically detects markedly increased levels of p53 protein in cell lines and tumors that bear mutant p53 $(24,25)$. Fig. $9 \mathrm{~A}$ demonstrates that Ab1801 shows strong reactivity with the $S W 480$ cell line, which bears a known p53 mutant (4), but that, as predicted, the p53 protein in the three VACO lines is present at only the barely detectable levels expected for wild type p53 protein. In comparison with Western blots, Northern blots detect equally both the wild type p53 transcript in the three VACO cell lines and the mutant p53 transcript in the SW480 cell line (Fig. $9 \mathrm{~B}$ ). Thus, as expected for cell lines bearing wild type p53 alleles, VACO-235 and VACO-411 express high levels of transcript but levels of protein well below those characteristic of mutant p53. Thus, both DNA sequencing and Western analysis demonstrate that the phenotypic progression of early passage VACO-235 to late passage VACO-235 and to VACO-411 proceeds via a pathway that does not require mutation or loss of the $\mathrm{p} 53$ gene product.

\section{Discussion}

We report the construction of a cell culture model of colon adenoma to colon carcinoma progression. This model has allowed us to examine the correlation between alterations in known colon cancer oncogenes and tumor suppressor genes with such features of phenotypic progression as acquisition of tumorigenicity, anchorage-independent growth, and alterations in growth factor control. Our observations include the resolution of colon neoplasia into a least three stages: a "be-
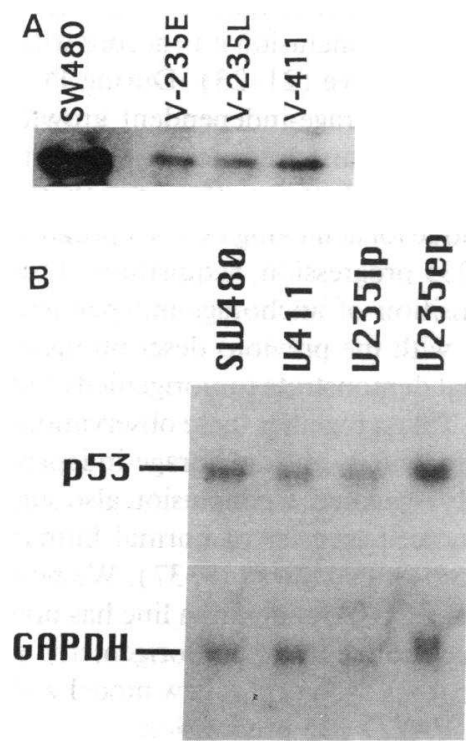

Figure 9. ( $A$ ) Expression of p 53 protein. Shown is a Western blot of $\mathrm{p} 53$ protein detected by antibody 1801 in extracts from the VACO-235 early passage $(V-235 E)$, VACO-235 late passage $(V-235 L)$, and VACO-411 cell line in comparison with mutant p53-bearing SW480 colon cancer cell line. $(B)$ Expression of p53 transcript. Shown is the autoradiogram of a Northern filter of RNA from VACO-235 early passage, VACO-235 late passage, VACO-411, and control line SW480, hybridized first to the p53 cDNA (top), then stripped and rehybridized to a control gene GAPDH ( bottom). nign" stage of anchorage-dependent, growth factor-dependent, nontumorigenic growth; a "malignant" stage of tumorigenic growth during which anchorage dependence and growth factor dependence are retained; and an "advanced" stage of high grade tumorigenicity accompanied by anchorage independence and growth factor independence. We find that absence of two colon cancer suppressor genes, by mutation of APC and by deletion and absent expression of DCC, along with activation of one colon cancer oncogene, by mutation of k-ras, in combination gives rise to the first stage of benign nontumorigenic behavior, as well as to a cell still fully responsive to exogenous positive and negative growth factors. We additionally establish that in our model progression to the "malignant" and "advanced" stages does not proceed by inactivation of the p53 suppressor gene. We suggest that in this model progression proceeds by a novel pathway that may be representative of that subset of colon cancers that retain wild type p53 alleles.

Our observations demonstrate that the combination of APC mutation, ras mutation, and absent DCC expression correlates in early passage VACO-235 with a "benign" phenotype. Specifically, we find the combination of absent DCC expression, ras mutation, and APC mutation is not in colon epithelium sufficient to confer any of: tumorigenicity, anchorage-independent growth, loss of epithelial differentiation, resistance to TGF-beta growth inhibition, or independence from serum or EGF growth stimulation. These observations are consistent with previous findings that ras mutation, deletion of one DCC allele, and deletion of one APC allele are detectable in tissue from one colon adenoma (2). This observation now further extends those findings by demonstrating that complete absence of DCC expression, previously described only in colon carcinomas, can occur at the colon adenoma stage. The mechanism for absent expression from the retained VACO-235 DCC allele remains uncharacterized, and could reflect either primary genetic alteration of the locus or epigenetic events such as those related to the state of cellular differentiation.

Escape from TGF-beta inhibition is suggested to be a key step during cancer progression (21), and DCC expression has been posited to play a role in mediating sensitivity to TGF-beta (13). Chromosome 18 transfer into the SW480 colon cancer cell line restores sensitivity of that cell line to inhibition by TGF-beta (13). Our observations, however, demonstrate that growth of early and late passage VACO-235 colon epithelial cells is strongly inhibited by TGF-beta, despite the absence of DCC expression in these cells. We cannot exclude that loss of DCC expression may have decreased sensitivity to TGF-beta of VACO-235 relative to normal colonic mucosa, or that DCC inactivation is a susceptibility event necessary for VACO-411's profound resistance to TGF-beta. However, progression to TGF-beta resistance in VACO-411 requires events additional to the loss of DCC expression. Our observations further demonstrate that K-ras mutation does not directly confer resistance to TGF-beta, at least not at a high level. This finding is compatible with previous observations that an independently derived colon adenoma cell line containing a mutant $\mathrm{K}$-ras allele is sensitive to TGF-beta (27), and that during mouse skin carcinogenesis resistance to TGF-beta occurs later than acquisition of ras mutations (28).

DCC has also been suggested to be a tumor suppressor gene functioning in part by being a cell adhesion molecule ( 3 ). This hypothesis is supported by DCC's sequence homology to neural cell adhesion molecule, and by the demonstration in 
Rat- 1 cells that antisense DCC expression both induces tumorigenicity and induces loss of cell adhesion to plastic $(3,29)$. Support for this model also derives from observations that transfer into the COKFu colon cancer cell line of an additional chromosome 18 , bearing a presumed wild type DCC allele, abolishes tumorigenicity, abolishes anchorage-independent growth, and confers a more epithelial appearance (30). Similar studies in the SW480 colon cancer cell line show that addition of a normal chromosome 18 diminishes the line's tumorigenicity without affecting either its morphology or capacity for anchorage-independent growth (13). Our data refine the understanding of DCC's function in maintenance of epithelial morphology and in possible tumor suppression. Despite absence of DCC expression, VACO-235 retains both epithelial morphology and apparent cell-to-cell adhesion. The further loss of epithelial morphology in culture seen in VACO-411 suggests that adhesion molecules additional to DCC must play key roles in the maintenance of the colonic epithelial morphology present in VACO-235. Our model demonstrates that combined APC mutation, ras mutation, and absence of DCC expression is by itself insufficient in colon epithelium to confer tumorigenicity, anchorage-independent growth, or loss of typical epithelial morphology. Loss of DCC expression of course may still be necessary for any of these phenotypes to emerge.

Unexpectedly, we find the easily detectable VACO-411 p53 transcript is wild type. In collaboration with Vogelstein and colleagues (4), we have previously reported that mutations were not detected in the coding regions of p53 genes from about one-third of human colon tumors when examined by sequencing PCR-amplified p53 exons 5-9 obtained from genomic DNA. It of course was possible that in these tumors p53 expression was inactivated by lesions in promoters or in splice junctions, areas not examined by sequencing the conserved p53 exons. We now demonstrate that the malignant VACO411 both retains and actively expresses wild type p53. Thus, VACO-411 may well represent an alternate pathway of colon cancer progression used by that subset of colon cancers that retain only wild type p53 alleles. Other investigators have recently confirmed our original observation, that a subset of colon cancers seem to bear only wild type p53 genes (31). Recently, Levine and colleagues (32) have reported that a subset of inflammatory breast carcinomas actively express wild type p53 protein. Additionally, Vogelstein and colleagues (33) have recently demonstrated that soft tissue sarcomas express wild type p53, which in this malignancy is inactivated by overexpression of an amplified MDM2 gene. All three cell lines, early and late passage VACO-235, and VACO-411, express MDM2 at an identical level, which is also equal to that found in normal colon mucosa (S. Markowitz, data not shown). Thus, MDM2 is unlikely to be involved in the phenotypic progression described in this model. It is certainly tempting to posit that progression to VACO-411 may involve alteration of an alternate genetic element that might interact with $\mathrm{p} 53$. Consistent with such a hypothesis is our previous report that an overexpressed transfected wild type p53 allele does not growth inhibit late passage VACO-235 (10). While we cannot at this juncture identify the mechanism of progression from VACO-235 to VACO-411, we suggest VACO-411 may be of value in identifying a novel pathway of colon cancer progression used by those colonic neoplasms that retain wild type p53 alleles.

Our observations are consistent with previous suggestions that colon carcinoma is a multistep process (34), resolvable in this model into stages of early passage VACO-235, late passage VACO-235, and VACO-411. The common origin of these lines is established both by common marker chromosomes and by the presence of the same mutant K-ras allele. We suggest the weak tumorigenicity of late passage VACO-235 reflects the occurrence in culture of a step permissive for progression to tumorigenic growth in the nude mouse. We suggest the phenotype recovered in VACO-411 reflects the selection under conditions of in vivo growth of additional event or events. While in vivo growth could potentially select for events not assayable in cell culture, such as increased angiogenesis or invasiveness, we note that VACO-411 has in fact acquired a new cassette of behaviors, including increased tumorigenicity, loss of anchorage dependence, and loss of sensitivity to both positive and negative growth factors. The association of these behaviors in a single model of progression suggests they may be etiologically linked to a common progression event. This hypothesis is supported by previous observations suggesting that loss of response to positive growth factors, including serum and EGF, distinguishes between independent colon cancer cell lines representing increasingly aggressive stages of the disease (22). This hypothesis is additionally supported by previous observations that loss of responsiveness to TGF-beta growth inhibition is also a general feature of malignant progression (21), distinguishes high grade from low grade colon cancer cell lines (22), and when induced can directly enhance tumorigenicity of one target colon cancer cell line (23).

The findings in the VACO-235/411 model contrast with previous observations made in studies of PC/ AA, a colon adenoma cell line established from an adenoma from a familial polyposis patient $(27,35)$. Progression of the PC/AA adenoma line to tumorigenicity was initially achieved by sequential treatment with butyrate, followed by exposure to the mutagen $N$-methyl- $N$-nitro- $N$-nitrosoguanidine, by passage through soft agar, and by an additional period of maintenance in cell culture (35). In contrast, VACO-235 was established from a sporadic adenoma, and progression occurred spontaneously under conditions of first growth in culture and then growth in the nude mouse (8). In the case of PC/AA initial tumorigenicity was accompanied by resistance to TGF-beta $(27,35)$. In the case of VACO-235 acquisition of tumorigenicity preceded acquisition of TGF-beta resistance, suggesting that resistance to TGF-beta is a distinct progression event that follows initial acquisition of tumorigenicity. This conclusion is in accord with previous observations discussed above (21-23). During PC/ AA progression capacity for anchorage-independent growth was acquired before acquisition of tumorigenicity. However, the capacity for anchorage-independent growth was required by its use as a selection technique for achieving PC/ AA progression. In the case of VACO-235 progression, acquisition of tumorigenicity preceded acquisition of anchorage-independent growth, a pattern consistent with the previous descriptions of two colon cancer cell lines that demonstrate tumorigenicity but not soft agar growth $(9,36)$. Taken together these observations reasonably suggest that tumorigenicity and anchorage-independent growth can be separately regulated, a conclusion also suggested by the results of microcell transfer of normal human chromosomes into human cancer cell lines $(13,37)$. We note the more recent report that the PC/AA adenoma line has now demonstrated spontaneous acquisition of tumorigenicity at passage 85 (38), and await comparison of this new model with the events described during VACO-235 progression. 
As our observations illustrate, cell culture models of colon cancer progression provide systems that are likely to be useful in elucidating the biologic consequences of genetic alterations currently known to be associated with colon cancer. Equally important, such models serve to illuminate facets of the colon cancer phenotype whose explanation may lay in the description of yet additional altered colon cancer genes.

\section{Acknowledgments}

We thank Dr. Bert Vogelstein for his kind gift of the MDM2 cDNA and for his assistance with establishing the methods for p53 cloning and sequencing. We thank Drs. Bert Vogelstein and Kathleen Cho for their assistance with establishing the method for DCC RT-PCR. We thank Drs. John Coffin, Jonathan Stoye, and Christopher Thomas for their kind gift of murine retroviral probes.

This study was supported by National Institutes of Health grants R29 CA-51504 to S. D. Markowitz, RO1 CA-57208-01 to S. D. Markowitz, and J. K. V. Willson, and P30 CA-43703 to Case Western Reserve University. L. Myeroff received support from Public Health Service award T32 HL-07147.

\section{References}

1. Sugarbaker, P., L. Gunderson, and R. Wittes. 1985. Colorectal cancer. In Cancer. Principles and Practice of Oncology. V. DeVita, S. Hellman, and S. Rosenberg, editors. J. B. Lippincott Company, Philadelphia. 795-884.

2. Vogelstein, B., E. Fearon, S. Hamilton, S. Kern, A. Presinger, M. Leppert, Y. Nakamura, R. White, A. Smits, and J. Bos. 1988. Genetic alterations during colorectal-tumor development. $N$. Engl. J. Med. 319:525-532.

3. Fearon, E., K. Cho, J. Nigro, S. Kern, J. Simons, J. M. Ruppert, S. Hamilton, A. Preisinger, G. Thomas, K. Kinzler, and B. Vogelstein. 1990. Identification of a chromosome 18q gene that is altered in colorectal cancers. Science (Wash. DC). 247:49-56.

4. Baker, S., A. Preisinger, J. M. Jessup, C. Paraskeva, S. Markowitz, J. K. V. Willson, S. Hamilton, and B. Vogelstein. 1990. p53 gene mutations occur in combination with $17 \mathrm{p}$ allelic deletions as late events in colorectal tumorigenesis. Cancer Res. 50:7717-7722.

5. Nishisho, I., Y. Nakamura, Y. Miyoshi, Y. Miki, H. Ando, A. Horii, K. Koyama, J. Utsunomiya, S. Baba, P. Hedge, et al. 1991. Mutations of chromosome 5q21 genes in FAP and colorectal cancer patients. Science (Wash. DC). 253:665-669.

6. Smith, K., K. Johnson, T. Bryan, D. Hill, S. Markowitz, J. Willson, C. Paraskeva, G. Petersen, S. Hamilton, B. Vogelstein, and K. Kinzler. 1993. The APC gene product in normal and tumor cells. Proc. Natl. Acad. Sci. USA. 90:2846-2850.

7. Powell, S., N. Zilz, Y. Beazer-Barclay, T. Bryan, S. Hamilton, S. Thibodeau, B. Vogelstein, and K. Kinzler. 1992. APC occur early during colorectal tumorigenesis. Nature (Lond.). 359:235-237.

8. Willson, J., G. Bittner, T. Oberley, G. Meisner, and J. Weese. 1987. Cell culture of human colon adenomas and carcinomas. Cancer Res. 47:2704-2713.

9. McBain, J., J. Weese, L. Meisner, W. Wolberg, and J. Willson. 1984. Establishment and characterization of human colorectal cancer cell lines. Cancer Res. 44:5813-5821.

10. Baker, S., S. Markowitz, E. Fearon, J. Willson, and B. Vogelstein. 1990. Suppression of human colorectal carcinoma cell growth by wild-type p 53 . Science (Wash. DC). 249:912-915.

11. Markowitz, S., K. Molkentin, C. Gerbic, J. Jackson, T. Stellato, and J. Willson. 1990. Growth stimulation by coexpression of transforming growth factor- $\alpha$ and epidermal growth factor-receptor in normal and adenomatous human colon epithelium. J. Clin. Invest. 86:356-362.

12. Suzuki, Y., M. Orita, M. Shiraishi, K. Hayashi, and T. Sekiya. 1990. Detection of ras gene mutations in human lung cancers by single-strand conformation polymorphism analysis of polymerase chain reaction products. Oncogene. 5:1037-1043.

13. Goyette, M., K. Cho, C. Fasching, D. Levy, K. Kinzler, C. Paraskeva, B. Vogelstein, and E. Stanbridge. 1992. Progression of colorectal cancer is associated with multiple tumor suppressor gene defects but inhibition of tumorigenicity is accomplished by correction of any single defect via chromosome transfer. Mol. Cell. Biol. 12:1387-1395.

14. Nigro, J., S. Baker, A. Preisinger, J. Jessup, R. Hostetter, K. Cleary, S. Bigner, N. Davidson, S. Baylin, P. Devilee, et al. 1989. Mutations in the p53 gene occur in diverse human tumour types. Nature (Lond.). 342:705-708.
15. Markowitz, S., G. Krystal, A. Lebacq-Verheyden, J. Way, E. Sausville, and J. Battey. 1988. Transcriptional activation and DNase I hypersensitive sites are associated with selective expression of the gastrin-releasing peptide gene. $J$. Clin. Invest. 82:808-815.

16. Feinberg, A., and B. Vogelstein. 1983. A technique for radiolabeling DNA restriction fragments to high specific activity. Anal. Biochem. 132:6-13.

17. Dugaiczyk, A., J. Haron, E. Stone, O. Dennison, K. Rothblum, and R. Schwartz. 1983. Cloning and sequencing of a deoxyribonucleic acid copy of glyceraldehyde-3-phosphate dehydrogenase messenger ribonucleic acid isolated from chicken muscle. Biochemistry. 22:1605-1613.

18. Davis, L., M. Dibner, and J. Battey. 1986. Basic Methods in Molecular Biology. Elsevier Science, Publishing Co., Inc., New York. 388 pp.

19. Massey, A., M. Coppola, and C. Thomas. 1990. Origin of pathogenic determinants of recombinant murine leukemia viruses: analysis of Bxv-1-related xenotropic viruses from CWD mice. J. Virol. 64:5491-5499.

20. Stoye, J., and J. Coffin. 1987. The four classes of endogenous murine leukemia virus: structural relationships and potential for recombination. J. Virol. 61:2659-2669.

21. Filmus, J., and R. Kerbel. 1993. Development of resistance mechanisms to the growth-inhibitory effects of transforming growth factor- $\beta$ during tumor progression. Curr. Opin. Oncol. 5:123-129.

22. Mulder, K., and M. Brattain. 1989. Growth factor expression and response in human colon carcinoma cells. In Cell and Molecular Biology of Colon Cancer. L. Augenlicht, editor. CRC Press, Inc., Boca Raton. 45-67.

23. Wu, S., D. Theodorescu, R. Kerbel, J. Willson, K. Mulder, L. Humphrey, and M. Brattain. 1992. TGF-beta 1 is an autocrine-negative growth regulator of human colon carcinoma FET cells in vivo as revealed by transfection of an antisense expression vector. J. Cell Biol. 116:187-196.

24. Hinds, P., C. Finlay, R. Quartin, S. Baker, E. Fearon, B. Vogelstein, and A. Levine. 1990. Mutant p53 dna clones from human colon carcinomas cooperate with ras in transforming primary rat cells: a comparison of the "hot spot" mutant phenotypes. Cell Growth \& Differ. 1:571-580.

25. Thor, A., D. Moore, S. Edgerton, E. Kawasaki, E. Reihsaus, H. Lynch, M Schwartz, L.-C. Chen, B. Mayall, and H. Smith. 1992. Accumulation of p53 tumor suppressor gene protein: an independent marker of prognosis in breast cancers. J. Natl. Cancer Inst. 84:845-855.

26. Iggo, R., K. Gatter, J. Bartek, D. Lane, and A. Harris. 1990. Increased expression of mutant forms of p53 oncogene in primary lung cancer. Lancet. 335:675-679.

27. Manning, A., A. Williams, S. Game, and C. Paraskeva. 1991. Differential sensitivity of human colonic adenoma and carcinoma cells to transforming growth factor $\beta$ (TGF $\beta$ ): conversion of an adenoma cell line to a tumorigenic phenotype is accompanied by a reduced response to the inhibitory effects of TGF- $\beta$. Oncogene. 6:1471-1476.

28. Haddow, S., D. Fowlis, K. Parkinson, R. Akhurst, and A. Balmain. 1991. Loss of growth control by TGF-beta occurs at a late stage of mouse skin carcinogenesis and is independent of ras gene activation. Oncogene. 6:1465-1470.

29. Narayanan, R., K. Lawlor, R. Schaapveld, K. Cho, B. Vogelstein, P. Tran, M. Osborne, and N. Telang. 1992. Antisense RNA to the putative tumor-suppressor gene DCC transforms Rat-1 fibroblasts. Oncogene. 7:553-561.

30. Tanaka, K., M. Oshimura, R. Kikuchi, M. Seki, T. Hayashi, and M. Miyaki. 1991. Suppression of tumorigenicity in human colon carcinoma cells by introduction of normal chromosome 5 or 18. Nature (Lond.). 349:340-342.

31. Ionov, Y., M. Peinado, S. Malkhosyan, D. Shibata, and M. Perucho. 1993. Ubiquitous somatic mutations in simple repeated sequences reveal a new mechanism for colonic carcinogenesis. Nature (Lond.). 363:558-561.

32. Moll, U., G. Riou, and A. Levine. 1992. Two distinct mechanisms alter p53 in breast cancer: Mutation and nuclear exclusion. Proc. Natl. Acad. Sci. USA 89:7262-7266

33. Oliner, J. D., K. Kinzler, P. Meltzer, D. Georges, and B. Vogelstein. 1992. Amplification of a gene encoding a p53-associated protein in human sarcomas. Nature (Lond.). 358:80-83.

34. Fearon, E., and B. Vogelstein. 1990. A genetic model for colorectal tumorigenesis. Cell. 61:759-767.

35. Williams, A., S. Harper, and C. Paraskeva. 1990. Neoplastic transformation of a human colonic epithelial cell line: in vitro evidence for the adenoma to carcinoma sequence. Cancer Res. 50:4724-4730.

36. Paraskeva, C., B. Buckle, D. Sheer, and C. Wigley. 1984. The isolation and characterization of colorectal epithelial cell lines at different stages in malignant transformation from familial polyposis coli patients. Int. J. Cancer. 34:49-56.

37. Weissman, B., P. Saxon, S. Pasquale, G. Jones, A. Geiser and E. Stanbridge. 1987. Introduction of a normal human chromosome 11 into a Wilms tumor cell line controls its tumorigenic expression. Science (Wash. DC). 236:175-180.

38. Paraskeva, C., A. Hague, N. Rooney, A. Williams, S. Harper, K. Hanlon, R. Atkinson, and A. Corfield. 1992. A single human colonic adenoma cell line can be converted in vitro to both a colorectal adenocarcinoma and a mucinous carcinoma. Int. J. Cancer. 51:661-664. 